\title{
Article \\ Correlative Study between Personality Traits, Student Mental Skills and Educational Outcomes
}

\author{
Oussama Bouiri ${ }^{1, *}$, Said Lotfi ${ }^{2, *}$ and Mohammed Talbi ${ }^{1, *}$ \\ 1 Laboratory of Analytical Chemistry and Physico-Chemistry of Materials, Hassan II University of Casablanca, \\ Casablanca 20100, Morocco \\ 2 Multidisciplinary Laboratory in Educations Sciences and Training Engineering, \\ Assessment in Sport Sciences and in Physical Activity Didactic, Normal Superior School (ENS), \\ Hassan II University of Casablanca, Casablanca 50069, Morocco \\ * Correspondence: oussama.bouiri-etu@etu.univh2c.ma (O.B.); SAID.LOTFI@univh2c.ma (S.L.); \\ mohammed.talbi@univh2m.ma (M.T.)
}

\begin{abstract}
The main purpose of this study is determining the correlations between personality traits, academic mental skills and educational outcomes using a quantitative methodology, based on a nonexperimental, correlational study. In addition, the following variables are taken into consideration: gender, grade averages and school cycle. The sample is composed of 695 students: these are two institutions (middle and high school) under the provincial direction of Mediouna. In order to gather the information, participants were asked to complete the 16pf 5 questionnaire and scale measuring academic mental skills. Note that the results obtained are processed by the IBM SPSS 23 software. The results demonstrate that the 16 personality scales of the Cattell 16PF5 test and the 9 school grades have significant correlations: $77.77 \%$ of all correlations, with essentially the following factors: abstractedness, tension, emotional stability, dominance, social-boldness, vigilance and apprehension maintain (8/9) significant and positive relationships with $38.88 \%$ and low intensity $(r=0.031$ to 0.0465). Additionally, mental skills (affective, cognitive and metacognitive strategies) and grades have significant correlations with $70.37 \%$ of all calculated correlations, with low, average and/or positive, negative intensities, according to each strategy with each grade of school subjects. In the end, it is necessary to make considerable efforts to better understanding the multidimensionality of school success and to ensure an effective and relevant pedagogical intervention.
\end{abstract}

Keywords: educational outcomes; correlation; student; learning strategies; personality traits

Academic Editor: Eila Jeronen

Received: 22 February 2021

Accepted: 12 March 2021

Published: 29 March 2021

Publisher's Note: MDPI stays neutral with regard to jurisdictional claims in published maps and institutional affiliations.

\section{Introduction}

In the act of teaching and learning centered on the development of the learner's skills as regards knowledge, the involvement of the learner is essential. Bandura (2007) [1] states that within the primary cycle the ability to organize cognitive, behavioral and emotional social skills to achieve a goal (learning tasks) promotes a sense of self-efficiency and eventually the success of the student in school.

Previous studies by Alexander, Entwisle, and Dauber (1993) [2], Florin, Guimard, and Nocus (2002) [3], Guimard, Cosnefroy, Florin (2007) [4], McClelland, Morrison, and Holmes (2000), (2006) [5,6], or Yen, Konold, and McDermott (2004) [7], have shown that students' performances are linked with their ability to organize and adapt to the teacher's instructions, while ensuring their participation and attention in the classroom. In addition, other studies conducted by Chamarro-Premuzic and Furnham (2003) [8] and Furnham, Chamorro-Premuzic, and McDougall (2002) [9] revealed that personality traits such as sensitivity, adaptation, independence, introversion and persistence would explain school performances variations in primary school. However, a study among the 15-16-year-old students has found that personality traits have a negative impact on their academic outcomes such as extraversion and emotional stability (Poropat, 2009) [10]. Moreover, at the University, personality traits help to explain the success or the failure of the students $[8,9]$. 
School performances depend not only on the student's personality traits, but also on his or her mental skills. Studies by Loret [11], Barca-Lozano, Peralbo, Almeida, Brenlla, Vicente, Porto, and Morán [12], or Tejedor-Tejedor, González, and García [13] have all demonstrated the relationship between learning strategies and school outcomes. A successful student is the one who can choose and use the right strategy that will enable him to reach the task needs [14-20].

Indeed, gender is generally viewed as one of many parameters that make a difference in using learning strategies. Further, there is also a strong competitive angle: the fact that women often perform higher than men in reading is consistent with the results of international studies [21,22].

Morocco has always been one of the countries on the lookout for new scientific developments in relation to the subjects of education and teaching as a whole. The "2015-2030" strategic vision is a practical example of the effort made by the Moroccan government, which has highlighted as one of its objectives: "Achieving sustainable development, in particular, by recognizing the right to education and modern education, of good quality and easy access" [23]. Accordingly, the purpose of this research is to study the correlation between the psychological characteristics of student's behavior, which are reflected in personality traits and mental skills, and its academic results for an effective and relevant educational intervention.

\section{Materials and Methods}

\subsection{Participants}

We carried out an exploratory survey of a sample of 695 students: these are two institutions under the provincial direction of Mediouna (the provincial direction of Mediouna: it is an administrative district, independent but linked to the Casablanca-Settat Academy) that made it possible to carry out our experiment. For the middle-school: 469 pupils in which $33.2 \%$ are male and $34.2 \%$ female. The high-school has 226 pupils: $12.9 \%$ male and $19.6 \%$ female. They have been accessed through random sampling, contacting the professors before proceeding with the data collection, in order to know their availability and the degree of agreement with the study's purpose. Table 1 below shows the characteristics of the participants of the study.

Table 1. Percentage of pupils by gender and school cycle.

\begin{tabular}{|c|c|c|c|c|c|c|c|}
\hline & \multirow{3}{*}{ Class } & \multicolumn{6}{|c|}{ Gender } \\
\hline & & \multicolumn{2}{|c|}{ Female } & \multicolumn{2}{|c|}{ Male } & \multicolumn{2}{|c|}{ Total } \\
\hline & & Workforce & $\mathbf{N} \%$ table & Workforce & N \% table & Workforce & $\mathbf{N} \%$ table \\
\hline \multirow{4}{*}{$\begin{array}{l}\text { Middle } \\
\text { school }\end{array}$} & 1st grade & 77 & $11.1 \%$ & 81 & $11.7 \%$ & 158 & $22.7 \%$ \\
\hline & 2nd grade & 83 & $11.9 \%$ & 64 & $9.2 \%$ & 147 & $21.2 \%$ \\
\hline & 3rd grade & 78 & $11.2 \%$ & 86 & $12.4 \%$ & 164 & $23.6 \%$ \\
\hline & Thotal & 238 & $34.2 \%$ & 231 & $33.2 \%$ & 469 & $67.5 \%$ \\
\hline \multirow{4}{*}{ High school } & 1st grade & 67 & $9.6 \%$ & 30 & $4.3 \%$ & 97 & $14.0 \%$ \\
\hline & 2nd grade & 29 & $4.2 \%$ & 37 & $5.3 \%$ & 66 & $9.5 \%$ \\
\hline & 3rd grade & 40 & $5.8 \%$ & 23 & $3.3 \%$ & 63 & $9.1 \%$ \\
\hline & Total & 136 & $19.6 \%$ & 90 & $12.9 \%$ & 226 & $32.5 \%$ \\
\hline \multicolumn{2}{|c|}{ Total } & 374 & $53.8 \%$ & 321 & $46.2 \%$ & 695 & $100.0 \%$ \\
\hline
\end{tabular}

The average age of our population is 14.87 years old. At the middle school, the boys' average is about 14.04 years old while the girls' average is about 13.91 years old (Table 2). In the high school, the average age is around 16.74 years old (16.66 years old for boys and 16.80 years old for girls) (Table 2). 
Table 2. Average age by gender and school cycle.

\begin{tabular}{|c|c|c|c|c|c|c|c|}
\hline & \multirow{4}{*}{ Class } & \multicolumn{6}{|c|}{ Gender } \\
\hline & & \multicolumn{2}{|c|}{ Female } & \multicolumn{2}{|c|}{ Male } & \multicolumn{2}{|c|}{ Total } \\
\hline & & \multicolumn{2}{|c|}{ Age } & \multicolumn{2}{|c|}{ Age } & \multicolumn{2}{|c|}{ Age } \\
\hline & & Average & $\begin{array}{l}\text { Standard } \\
\text { Deviation }\end{array}$ & Average & $\begin{array}{l}\text { Standard } \\
\text { Deviation }\end{array}$ & Average & $\begin{array}{l}\text { Standard } \\
\text { Deviation }\end{array}$ \\
\hline \multirow{4}{*}{$\begin{array}{l}\text { Middle } \\
\text { school }\end{array}$} & 1st grade & 12.86 & 1.13 & 13.02 & 1.29 & 12.94 & 1.22 \\
\hline & 2nd grade & 13.71 & 0.97 & 13.80 & 0.98 & 13.75 & 0.97 \\
\hline & 3rd grade & 15.15 & 1.15 & 15.19 & 1.25 & 15.17 & 1.20 \\
\hline & Total & 13.91 & 1.43 & 14.04 & 1.51 & 13.97 & 1.47 \\
\hline \multirow{4}{*}{ High school } & 1st grade & 15.94 & 0.78 & 15.73 & 0.74 & 15.88 & 0.77 \\
\hline & 2nd grade & 16.93 & 0.75 & 16.73 & 0.69 & 16.82 & 0.72 \\
\hline & 3rd grade & 18.15 & 1.14 & 17.74 & 0.92 & 18.00 & 1.08 \\
\hline & Total & 16.80 & 1.30 & 16.66 & 1.08 & 16.74 & 1.22 \\
\hline \multicolumn{2}{|c|}{ Total } & 14.96 & 1.97 & 14.78 & 1.83 & 14.87 & 1.91 \\
\hline
\end{tabular}

\subsection{Instruments}

- Test 16 PF5: The Fifth Edition of the Questionnaire 16 PF 5 Authors: RB Cattell, AK Cattell and Cattell. Heather, E. P (1993) aims to assess the first 16-order characteristics and five overall dimensions of personality. He identified 12 personality traits. Its aim is to provide a rich and nuanced description of the personality sphere. The description of the behaviors can be summarized in 5 dimensions: extraversion, independence, self-control, anxiety, tough-mindedness.

- Scale measuring academic mental skills: We designed this scale to measure the three learning strategies. It consists of 72 statements, each of which concerns learning strategies: affective strategies whose criteria are: (self-criticism, emotional management, distrust, curiosity, vigilance in school, pleasure, motivation, determination and communication), cognitive strategies (analysis, understanding, concentration, differentiation, effectiveness, and metacognitive strategies (effective policy research, inhibition, organization, time planning, and cognitive regulation). This scale has 5 inverted items. It corresponds to specific periods (at school, at home, etc.). For measuring scales, the Likert scale was chosen.

- School results: Student's grades were retrieved from the "MASSAR "platform (the "MASSAR "platform is a school management system adopted by the Moroccan Ministry of national education.). These are 8 grades of school subjects as well as the general average.

\subsection{Design}

A quantitative approach is used: an empirical and correlational study that analyzes personality traits, mental skills and their relationship with educational success. We administered the tests to students from different ages and grades at the same time. We note that results may lead to a new orientation in the pedagogical reflection.

\subsection{Data Analysis}

The analyses of the relationship between the 16 personality factors, mental skills and the 9 school grades was made by Bravais Pearson. Correlations analyses are presented in the form of correlation matrices ( $\mathrm{N}, \mathrm{p}, \mathrm{r}, \mathrm{r} 2)$. Partial correlations were used to examine all these associations (controlling for age, gender and school cycle). They are presented on average and standard deviations. The threshold of significance is set $(p=0.05)$. The data were processed by SPSS 23 IBM. IC. CHICAGO. 


\section{Results}

\subsection{Correlation between Personality Traits and Academic Outcomes}

There is a correlation between the 16 personality traits included in the 16 pf5 test and each grade of the school subjects as well as the grade averages, controlled by these three variables: the grade averages, gender and school cycle. The 16 personality dimensions have highly significant correlations with the averages score, low intensity (liveliness, privateness, abstractedness, openness to change and vigilance) and medium (warmth, rule-consciousness, self-reliance, tension, emotional stability, dominance, social-boldness, sensitivity, apprehension, perfectionism and reasoning).

Table 3, presents the coefficient of correlation « $\mathrm{r}$ » of the grade averages, as well as the grades of 8 school subjects with the 16 dimensions of personality according to 16 pf5 test. We found that of the 128 correlations calculated, 96 relationships were positive, very significant ( $p=0.009$ to 0.01 ), or $75 \%$ of all relationships. We note that privateness and vigilance have highly significant correlations $(p=0.00)$. These correlations are explained by the control effect on the inter-trait partial correlations, which have the average of the grades, the gender and the school cycle.

Table 3. Summary of the partial correlations between personality traits controlled by the grade averages, gender, and school cycle (middle school, high school).

\begin{tabular}{|c|c|c|c|c|c|}
\hline & \multicolumn{2}{|c|}{ Coefficient of $r$} & \multicolumn{3}{|c|}{ Control Effect on Inter-Trait Partial } \\
\hline & $\begin{array}{l}\text { Grade } \\
\text { Averages }\end{array}$ & $\begin{array}{c}\text { Number of } \\
\text { Materials } \\
(p<0.05)\end{array}$ & $\begin{array}{l}\text { Grade Averages } \\
(p<0.05)\end{array}$ & Gender & $\begin{array}{l}\text { School Cycle (Middle } \\
\text { School-High School) }\end{array}$ \\
\hline Warmth & $0.340^{* *}$ & $6 / 8$ & $\begin{array}{l}\text { Rule-consciousness, } \\
\text { Abstractedness, } \\
\text { Social-boldness, } \\
\text { Sensitivity, } \\
\text { Perfectionism, } \\
\text { Reasoning. }\end{array}$ & $\begin{array}{c}\text { Rule-consciousness, } \\
\text { Abstractedness, } \\
\text { Self-reliance, Tension, } \\
\text { Emotional stability, } \\
\text { Dominance, } \\
\text { Social-boldness, } \\
\text { Sensitivity, } \\
\text { Apprehension, } \\
\text { Perfectionism, } \\
\text { Reasoning. }\end{array}$ & $\begin{array}{c}\text { Rule-consciousness, } \\
\text { Abstractedness, } \\
\text { Dominance, } \\
\text { Social-boldness, } \\
\text { Sensitivity, } \\
\text { Apprehension, } \\
\text { Perfectionism, } \\
\text { Reasoning. }\end{array}$ \\
\hline Liveliness & $0.172 * *$ & $6 / 8$ & $\begin{array}{c}\text { Privateness, } \\
\text { Openness to change, } \\
\text { Vigilance, } \\
\text { Perfectionism, }\end{array}$ & $\begin{array}{l}\text { Privateness, Openness to } \\
\text { change, Self-reliance, } \\
\text { Tension, Emotional } \\
\text { stability, Sensitivity, } \\
\text { Vigilance, Apprehension, } \\
\text { Perfectionism, } \\
\text { Reasoning. }\end{array}$ & $\begin{array}{c}\text { Privateness, Openness } \\
\text { to change, Vigilance, } \\
\text { Perfectionism. }\end{array}$ \\
\hline $\begin{array}{c}\text { Rule- } \\
\text { Consciousness }\end{array}$ & $0.310^{* *}$ & $5 / 8$ & $\begin{array}{l}\text { Warmth, } \\
\text { Abstractedness, } \\
\text { Self-reliance, } \\
\text { Dominance, } \\
\text { Social-boldness, } \\
\text { Sensitivity, } \\
\text { Apprehension, } \\
\text { Perfectionism, } \\
\text { Reasoning }\end{array}$ & $\begin{array}{l}\text { Warmth, Abstractedness, } \\
\text { Self-reliance, Tension, } \\
\text { Emotional stability, } \\
\text { Dominance, } \\
\text { Social-boldness, } \\
\text { Sensitivity, } \\
\text { Apprehension, } \\
\text { Perfectionism, } \\
\text { Reasoning. }\end{array}$ & $\begin{array}{c}\text { Warmth, } \\
\text { Abstractedness, } \\
\text { Self-reliance, Tension, } \\
\text { Dominance, } \\
\text { Social-boldness, } \\
\text { Sensitivity, } \\
\text { Apprehension, } \\
\text { Perfectionism, } \\
\text { Reasoning. }\end{array}$ \\
\hline Privateness & $0.117^{* * *}$ & $3 / 8$ & $\begin{array}{c}\text { Liveliness, Openness } \\
\text { to change, Vigilance, } \\
\text { Apprehension, } \\
\text { Perfectionism, }\end{array}$ & $\begin{array}{l}\text { Liveliness, Openness to } \\
\text { change, Tension, } \\
\text { Emotional stability, } \\
\text { Vigilance, Apprehension, } \\
\text { Perfectionism, } \\
\text { Reasoning. }\end{array}$ & $\begin{array}{l}\text { Liveliness, Openness } \\
\text { to change, Vigilance, } \\
\text { Apprehension, } \\
\text { Perfectionism, } \\
\text { Reasoning. }\end{array}$ \\
\hline
\end{tabular}


Table 3. Cont.

\begin{tabular}{|c|c|c|c|c|c|}
\hline & \multicolumn{2}{|c|}{ Coefficient of $r$} & \multicolumn{3}{|c|}{ Control Effect on Inter-Trait Partial } \\
\hline & $\begin{array}{c}\text { Grade } \\
\text { Averages }\end{array}$ & $\begin{array}{c}\text { Number of } \\
\text { Materials } \\
(p<0.05)\end{array}$ & $\begin{array}{l}\text { Grade Averages } \\
(p<0.05)\end{array}$ & Gender & $\begin{array}{l}\text { School Cycle (Middle } \\
\text { School-High School) }\end{array}$ \\
\hline Abstractedness & $0.259 * *$ & $7 / 8$ & $\begin{array}{c}\text { Warmth, } \\
\text { Rule-consciousness, } \\
\text { Self-reliance, } \\
\text { Emotional stability, } \\
\text { Dominance, } \\
\text { Social-boldness, } \\
\text { Sensitivity, } \\
\text { Apprehension, } \\
\text { Perfectionism, } \\
\text { Reasoning. }\end{array}$ & $\begin{array}{l}\text { Warmth, } \\
\text { Rule-consciousness, } \\
\text { Openness to change, } \\
\text { Self-reliance, Tension, } \\
\text { Vigilance, Emotional } \\
\text { stability, Dominance, } \\
\text { Social-boldness, } \\
\text { Sensitivity, } \\
\text { Apprehension, } \\
\text { Perfectionism, } \\
\text { Reasoning. }\end{array}$ & $\begin{array}{c}\text { Warmth, } \\
\text { Rule-consciousness, } \\
\text { Self-reliance, Tension, } \\
\text { Emotional stability, } \\
\text { Dominance, } \\
\text { Social-boldness, } \\
\text { Sensitivity, } \\
\text { Apprehension, } \\
\text { Perfectionism, } \\
\text { Reasoning. }\end{array}$ \\
\hline $\begin{array}{l}\text { Openness to } \\
\text { change }\end{array}$ & $0.145^{* *}$ & $5 / 8$ & $\begin{array}{c}\text { Liveliness, } \\
\text { Privateness, Tension, } \\
\text { Dominance, } \\
\text { Vigilance, } \\
\text { Apprehension, } \\
\text { Perfectionism. }\end{array}$ & $\begin{array}{l}\text { Liveliness, Privateness, } \\
\text { Abstractedness, Tension, } \\
\text { Emotional stability, } \\
\text { Dominance, Sensitivity, } \\
\text { Vigilance, Apprehension, } \\
\text { Perfectionism, } \\
\text { Reasoning. }\end{array}$ & $\begin{array}{c}\text { Liveliness, Privateness, } \\
\text { Tension, Dominance, } \\
\text { Vigilance, } \\
\text { Apprehension, } \\
\text { Reasoning. }\end{array}$ \\
\hline Self-Reliance & $0.333 * *$ & $6 / 8$ & $\begin{array}{l}\text { Rule-consciousness, } \\
\text { Abstractedness, } \\
\text { Tension, Emotional } \\
\text { stability, Dominance, } \\
\text { Social-boldness, } \\
\text { Sensitivity, } \\
\text { Apprehension, } \\
\text { Perfectionism, } \\
\text { Reasoning. }\end{array}$ & $\begin{array}{l}\text { Warmth, Liveliness, } \\
\text { Rule-consciousness, } \\
\text { Abstractedness, Tension, } \\
\text { Vigilance, Emotional } \\
\text { stability, Dominance, } \\
\text { Social-boldness, } \\
\text { Sensitivity, } \\
\text { Apprehension, } \\
\text { Perfectionism, } \\
\text { Reasoning. }\end{array}$ & $\begin{array}{c}\text { Rule-consciousness, } \\
\text { Abstractedness, } \\
\text { Tension, Emotional } \\
\text { stability, Dominance, } \\
\text { Sensitivity, } \\
\text { Apprehension, } \\
\text { Perfectionism, } \\
\text { Reasoning. }\end{array}$ \\
\hline Tension & $0.358^{* *}$ & $7 / 8$ & $\begin{array}{c}\text { Rule-consciousness, } \\
\text { Openness to change, } \\
\text { Abstractedness, } \\
\text { Self-reliance, } \\
\text { Emotional stability, } \\
\text { Dominance, } \\
\text { Social-boldness, } \\
\text { Sensitivity, } \\
\text { Apprehension, } \\
\text { Perfectionism, } \\
\text { Reasoning. }\end{array}$ & $\begin{array}{l}\text { Warmth, Liveliness, } \\
\text { Rule-consciousness, } \\
\text { Privateness, } \\
\text { Abstractedness, } \\
\text { Openness to change, } \\
\text { Self-reliance, Emotional } \\
\text { stability, Dominance, } \\
\text { Social-boldness, } \\
\text { Sensitivity, } \\
\text { Apprehension, } \\
\text { Perfectionism, } \\
\text { Reasoning. }\end{array}$ & $\begin{array}{c}\text { Rule-consciousness, } \\
\text { Abstractednesss, } \\
\text { Openness to change, } \\
\text { Self-reliance, } \\
\text { Emotional stability, } \\
\text { Dominance, Boldness, } \\
\text { Sensitivity, } \\
\text { Apprehension, } \\
\text { Perfectionism, } \\
\text { Reasoning. }\end{array}$ \\
\hline $\begin{array}{l}\text { Emotional } \\
\text { stability }\end{array}$ & $0.355^{* *}$ & $7 / 8$ & $\begin{array}{l}\text { Abstractedness, } \\
\text { Dominance, } \\
\text { Social-boldness, } \\
\text { Sensitivity, } \\
\text { Apprehension, } \\
\text { Perfectionism, } \\
\text { Reasoning. }\end{array}$ & $\begin{array}{l}\text { Warmth, Liveliness, } \\
\text { Rule-consciousness, } \\
\text { Privateness, } \\
\text { Abstractedness, } \\
\text { Openness to change, } \\
\text { Self-reliance, Tension, } \\
\text { Dominance, } \\
\text { Social-boldness, } \\
\text { Sensitivity, } \\
\text { Apprehension, } \\
\text { Perfectionism, } \\
\text { Reasoning. }\end{array}$ & $\begin{array}{c}\text { Abstractedness, } \\
\text { Self-reliance, Tension, } \\
\text { Dominance, } \\
\text { Social-boldness, } \\
\text { Apprehension, } \\
\text { Perfectionism, } \\
\text { Reasoning. }\end{array}$ \\
\hline
\end{tabular}


Table 3. Cont.

\begin{tabular}{|c|c|c|c|c|c|}
\hline & \multicolumn{2}{|c|}{ Coefficient of $\mathbf{r}$} & \multicolumn{3}{|c|}{ Control Effect on Inter-Trait Partial } \\
\hline & $\begin{array}{c}\text { Grade } \\
\text { Averages }\end{array}$ & $\begin{array}{c}\text { Number of } \\
\text { Materials } \\
(p<0.05)\end{array}$ & $\begin{array}{l}\text { Grade Averages } \\
(p<0.05)\end{array}$ & Gender & $\begin{array}{l}\text { School Cycle (Middle } \\
\text { School-High School) }\end{array}$ \\
\hline Dominance & $0.328^{* *}$ & $7 / 8$ & $\begin{array}{c}\text { Rule-consciousness, } \\
\text { Abstractedness, } \\
\text { Openness to change, } \\
\text { Self-reliance, } \\
\text { Tension, Emotional } \\
\text { stability, } \\
\text { Social-boldness, } \\
\text { Sensitivity, } \\
\text { Apprehension, } \\
\text { Perfectionism, } \\
\text { Reasoning. }\end{array}$ & $\begin{array}{c}\text { Warmth, } \\
\text { Rule-consciousness, } \\
\text { Abstractedness, } \\
\text { Openness to change, } \\
\text { Self-reliance, Tension, } \\
\text { Emotional stability, } \\
\text { Social-boldness, } \\
\text { Sensitivity, } \\
\text { Apprehension, } \\
\text { Perfectionism, } \\
\text { Reasoning. }\end{array}$ & $\begin{array}{c}\text { Warmth, } \\
\text { Rule-consciousness, } \\
\text { Abstractedness, } \\
\text { Openness to change, } \\
\text { Self-reliance, Tension, } \\
\text { Emotional stability, } \\
\text { Social-boldness, } \\
\text { Sensitivity, } \\
\text { Apprehension, } \\
\text { Perfectionism, } \\
\text { Reasoning. }\end{array}$ \\
\hline $\begin{array}{l}\text { Social- } \\
\text { Boldness }\end{array}$ & $0.342^{* *}$ & $7 / 8$ & $\begin{array}{c}\text { Warmth, } \\
\text { Rule-consciousness, } \\
\text { Abstractedness, } \\
\text { Emotional stability, } \\
\text { Dominance, } \\
\text { Sensitivity, } \\
\text { Apprehension, } \\
\text { Perfectionism, } \\
\text { Reasoning. }\end{array}$ & $\begin{array}{c}\text { Warmth, } \\
\text { Rule-consciousness, } \\
\text { Abstractedness, } \\
\text { Self-reliance, Tension, } \\
\text { Emotional stability, } \\
\text { Dominance, Sensitivity, } \\
\text { Vigilance, Apprehension, } \\
\text { Perfectionism, } \\
\text { Reasoning. }\end{array}$ & $\begin{array}{c}\text { Warmth, } \\
\text { Rule-consciousness, } \\
\text { Abstractedness, } \\
\text { Self-reliance, Tension, } \\
\text { Emotional stability, } \\
\text { Dominance, } \\
\text { Sensitivity, } \\
\text { Apprehension, } \\
\text { Perfectionism, } \\
\text { Reasoning. }\end{array}$ \\
\hline Sensitivity & $0.316^{* *}$ & $5 / 8$ & $\begin{array}{c}\text { Warmth, } \\
\text { Rule-consciousness, } \\
\text { Abstractedness, } \\
\text { Self-reliance, } \\
\text { Tension, Emotional } \\
\text { stability, Dominance, } \\
\text { Social-boldness, } \\
\text { Vigilance, } \\
\text { Apprehension, } \\
\text { Perfectionism, } \\
\text { Reasoning. }\end{array}$ & $\begin{array}{l}\text { Warmth, Liveliness, } \\
\text { Rule-consciousness, } \\
\text { Abstractedness, } \\
\text { Openness to change, } \\
\text { Self-reliance, Tension, } \\
\text { Emotional stability, } \\
\text { Dominance, } \\
\text { Social-boldness, } \\
\text { Apprehension, } \\
\text { Perfectionism, } \\
\text { Reasoning. }\end{array}$ & $\begin{array}{c}\text { Warmth, } \\
\text { Rule-consciousness, } \\
\text { Abstractedness, } \\
\text { Self-reliance, Tension, } \\
\text { Emotional stability, } \\
\text { Dominance, } \\
\text { Social-boldness, } \\
\text { Vigilance, } \\
\text { Apprehension, } \\
\text { Perfectionism, } \\
\text { Reasoning. }\end{array}$ \\
\hline Vigilance & $0.138^{* * *}$ & $7 / 8$ & $\begin{array}{c}\text { Liveliness, } \\
\text { Privateness, } \\
\text { Openness to change, } \\
\text { Sensitivity, } \\
\text { Perfectionism. }\end{array}$ & $\begin{array}{l}\text { Liveliness, Privateness, } \\
\text { Abstractedness, } \\
\text { Openness to change, } \\
\text { Emotional stability, } \\
\text { Apprehension, } \\
\text { Perfectionism, } \\
\text { Reasoning. }\end{array}$ & $\begin{array}{c}\text { Liveliness, Privateness, } \\
\text { Openness to change, } \\
\text { Sensitivity. }\end{array}$ \\
\hline Apprehension & $0.401^{* *}$ & $7 / 8$ & $\begin{array}{c}\text { Warmth, } \\
\text { Rule-consciousness, } \\
\text { Privateness, } \\
\text { Abstractedness, } \\
\text { Openness to change, } \\
\text { Self-reliance, } \\
\text { Tension, Emotional } \\
\text { stability, Dominance, } \\
\text { Social-Boldness, } \\
\text { Sensitivity, } \\
\text { Perfectionism, } \\
\text { Reasoning. }\end{array}$ & $\begin{array}{c}\text { Warmth, Liveliness, } \\
\text { Rule-consciousness, } \\
\text { Privateness, } \\
\text { Abstractedness, } \\
\text { Openness to change, } \\
\text { Self-reliance, Tension, } \\
\text { Emotional stability, } \\
\text { Dominance, } \\
\text { Social-boldness, } \\
\text { Sensitivity, Vigilance, } \\
\text { Perfectionism, } \\
\text { Reasoning. }\end{array}$ & $\begin{array}{c}\text { Warmth, } \\
\text { Rule-consciousness, } \\
\text { Privateness, } \\
\text { Abstractedness, } \\
\text { Openness to change, } \\
\text { Self-reliance, Tension, } \\
\text { Emotional stability, } \\
\text { Dominance, } \\
\text { Social-Boldness, } \\
\text { Sensitivity, } \\
\text { Perfectionism, } \\
\text { Reasoning. }\end{array}$ \\
\hline
\end{tabular}


Table 3. Cont.

\begin{tabular}{|c|c|c|c|c|c|}
\hline & \multicolumn{2}{|c|}{ Coefficient of $r$} & \multicolumn{3}{|c|}{ Control Effect on Inter-Trait Partial } \\
\hline & $\begin{array}{c}\text { Grade } \\
\text { Averages }\end{array}$ & $\begin{array}{c}\text { Number of } \\
\text { Materials } \\
(p<0.05)\end{array}$ & $\begin{array}{l}\text { Grade Averages } \\
\qquad(p<0.05)\end{array}$ & Gender & $\begin{array}{l}\text { School Cycle (Middle } \\
\text { School-High School) }\end{array}$ \\
\hline Perfectionism & $0.379^{* *}$ & $6 / 8$ & $\begin{array}{c}\text { Warmth, Liveliness, } \\
\text { Rule-consciousness, } \\
\text { Privateness, } \\
\text { Abstractedness, } \\
\text { Openness to change, } \\
\text { Self-reliance, } \\
\text { Tension, Emotional } \\
\text { stability, Dominance, } \\
\text { Social-boldness, } \\
\text { Sensitivity, } \\
\text { Apprehension, } \\
\text { Reasoning }\end{array}$ & $\begin{array}{l}\text { Warmth, Liveliness, } \\
\text { Rule-consciousness, } \\
\text { Privateness, } \\
\text { Abstractedness, } \\
\text { Openness to change, } \\
\text { Self-reliance, Tension, } \\
\text { Emotional stability, } \\
\text { Dominance, } \\
\text { Social-boldness, } \\
\text { Sensitivity, Vigilance, } \\
\text { Apprehension, } \\
\text { Reasoning. }\end{array}$ & $\begin{array}{c}\text { Warmth, Liveliness, } \\
\text { Rule-consciousness, } \\
\text { Privateness, } \\
\text { Abstractedness, } \\
\text { Openness to change, } \\
\text { Self-reliance, Tension, } \\
\text { Emotional stability, } \\
\text { Dominance, } \\
\text { Social-boldness, } \\
\text { Sensitivity, } \\
\text { Apprehension, } \\
\text { Reasoning }\end{array}$ \\
\hline Reasoning & $0.364^{* *}$ & $5 / 8$ & $\begin{array}{c}\text { Warmth, Liveliness, } \\
\text { Rule-consciousness, } \\
\text { Privateness, } \\
\text { Abstractedness, } \\
\text { Openness to change, } \\
\text { Self-reliance, } \\
\text { Tension, Emotional } \\
\text { stability, Dominance, } \\
\text { Social-boldness, } \\
\text { Sensitivity, } \\
\text { Apprehension, } \\
\text { Perfectionism, } \\
\text { Reasoning. }\end{array}$ & $\begin{array}{l}\text { Warmth, Liveliness, } \\
\text { Rule-consciousness, } \\
\text { Abstractedness, } \\
\text { Openness to change, } \\
\text { Self-reliance, Tension, } \\
\text { Emotional stability, } \\
\text { Dominance, } \\
\text { Social-boldness, } \\
\text { Sensitivity, Vigilance, } \\
\text { Perfectionism, } \\
\text { Reasoning. }\end{array}$ & $\begin{array}{c}\text { Warmth, } \\
\text { Rule-consciousness, } \\
\text { Abstractedness, } \\
\text { Self-reliance, Tension, } \\
\text { Emotional stability, } \\
\text { Dominance, } \\
\text { Social-boldness, } \\
\text { Sensitivity, } \\
\text { Apprehension, } \\
\text { Perfectionism. }\end{array}$ \\
\hline
\end{tabular}

${ }^{* * *}$ Highly significant $(p=0,00) ;{ }^{* *}$ Very significant $(p=0.009$ to 0.01$)$.

The most significant factors are: abstractedness, tension, emotional stability, dominance, social-boldness, vigilance and apprehension present almost all (7/8) significant correlations ( $51.04 \%$ all significant relationships). When controlled by grade averages, gender and school cycle, it is noted that the control effect on intermediate partial correlations traits is high for each trait except for emotional stability, which has an average result in terms of grade averages and school cycle, as well as vigilance, with an average effect for gender and low relative to grade averages and school cycle.

The following personality factors: warmth, liveliness, self-reliance and perfectionism $(6 / 8)$ have significant correlations with school grades for each $(25 \%$ of all significant relationships): When controlled by grade averages, the gender and school cycle, we realize that the control effect on the inter-trait partial correlations is medium or low for each line, except for perfectionism, which has a high result with regard to grades averages and school cycle.

The results for the following factors: rule-consciousness, openness to change, sensitivity and reasoning present (5/8) significant correlations with school grades for each $20.83 \%$ of the significant correlations found. When controlled by grade averages, gender and school cycle, it is noted that the control effects on intermediate partial correlations traits are high for each trait except for the rule-consciousness which has an average score in terms of grades. As well as openness to change to experience with an average effect compared to average grades and the school cycle.

The privateness factor presents (3/8) correlations with school results, which could be compared to a percentage of $3.12 \%$ with all significant correlations. When controlled by grade's averages, gender and school cycle, the control effect on inter-trait partial correlations is medium. 


\subsection{Correlation between Affective Strategies with Each Academic Outcome}

These maintain highly significant correlations with the grade averages except for determination and pleasure, with an average and positive intensity (acceptance of critics, autonomy, communication, school confidence, curiosity, distrust and motivation), and low, negative intensity for emotional management.

They concern the correlation between 10 parameters. It is remarkable that out of 80 correlations analyzed, 61 relationships are very significant ( $p=0.009$ to 0.01 ), or $76.25 \%$ of all significant relationships. Only communication had highly significant correlations $(p=0.00)$.

Table 4 presents the coefficient of correlation «r» of the grade averages, as well as the scores of 8 school subjects with the 10 emotional parameters, these correlations are explained by the control effect on the inter-trait partial correlations, which are the average of the grades, the gender, and the school cycle.

Table 4. Summary of the partial correlations between the affective skills controlled by the average effect of grades, gender, and school cycle (middle school, high school).

\begin{tabular}{|c|c|c|c|c|c|}
\hline & \multicolumn{2}{|c|}{ Coefficient of $r$} & \multicolumn{3}{|c|}{ Control Effect on Inter-Trait Partial } \\
\hline & $\begin{array}{l}\text { Grade } \\
\text { Averages }\end{array}$ & $\begin{array}{l}\text { Number of } \\
\text { Subjects } \\
(p<0.05)\end{array}$ & $\begin{array}{l}\text { Grade Averages } \\
\qquad(p<0.05)\end{array}$ & Gender & $\begin{array}{l}\text { School Cycle (Middle } \\
\text { School-High School) }\end{array}$ \\
\hline $\begin{array}{l}\text { Acceptance of } \\
\text { errors and critics }\end{array}$ & $0.273^{* *}$ & $8 / 8$ & $\begin{array}{c}\text { Autonomy, } \\
\text { Communication, } \\
\text { Curiosity, Motivation }\end{array}$ & $\begin{array}{l}\text { Communication, } \\
\text { Curiosity, }\end{array}$ & $\begin{array}{l}\text { Communication, } \\
\text { curiosity }\end{array}$ \\
\hline Autonomy & $0.501^{* *}$ & $8 / 8$ & $\begin{array}{l}\text { Acceptance of errors } \\
\text { and Critics, } \\
\text { Communication, } \\
\text { Distrust, Motivation, } \\
\text { Confidence at School }\end{array}$ & $\begin{array}{c}\text { Confidence at } \\
\text { School, Curiosity, } \\
\text { Distrust, Motivation }\end{array}$ & $\begin{array}{l}\text { Confidence at School, } \\
\text { Curiosity, Distrust }\end{array}$ \\
\hline Communication & $0.262^{* * *}$ & $7 / 8$ & $\begin{array}{l}\text { Acceptance of errors } \\
\text { and critics, Autonomy, } \\
\text { Curiosity, Distrust, } \\
\text { Motivation }\end{array}$ & $\begin{array}{c}\text { Acceptance of errors } \\
\text { and critics, } \\
\text { Confidence at } \\
\text { School, Curiosity, } \\
\text { Motivation }\end{array}$ & $\begin{array}{l}\text { Acceptance of errors } \\
\text { and Critics, } \\
\text { Confidence at School, } \\
\text { Curiosity. }\end{array}$ \\
\hline $\begin{array}{l}\text { Confidence at } \\
\text { school }\end{array}$ & $0.299 * *$ & $7 / 8$ & $\begin{array}{l}\text { Distrust, Emotions } \\
\text { management, }\end{array}$ & $\begin{array}{l}\text { Autonomy, Curiosity, } \\
\text { Distrust, Motivation }\end{array}$ & $\begin{array}{c}\text { Autonomy, } \\
\text { Communication, } \\
\text { Curiosity, Distrust, } \\
\text { Motivation }\end{array}$ \\
\hline Curiosity & $0.446^{* *}$ & $8 / 8$ & $\begin{array}{c}\text { Acceptance of Errors } \\
\text { and Critics, } \\
\text { Communication, } \\
\text { Emotions } \\
\text { management }\end{array}$ & $\begin{array}{l}\text { Acceptance of errors } \\
\text { and critics, } \\
\text { Autonomy, } \\
\text { Communication, } \\
\text { Confidence at school, } \\
\text { Distrust, Motivation }\end{array}$ & $\begin{array}{l}\text { Acceptance of errors } \\
\text { and critics, Autonomy, } \\
\text { Communication, } \\
\text { Confidence at school, } \\
\text { Distrust, Motivation. }\end{array}$ \\
\hline Distrust & $0.355^{* *}$ & $8 / 8$ & $\begin{array}{c}\text { Autonomy, } \\
\text { Communication, } \\
\text { Confidence at school, } \\
\text { Gestion of emotions, } \\
\text { Motivation }\end{array}$ & $\begin{array}{c}\text { Autonomy, } \\
\text { Confidence at school, } \\
\text { Curiosity, } \\
\text { Motivation }\end{array}$ & $\begin{array}{l}\text { Autonomy, } \\
\text { Confidence at school, } \\
\text { Curiosity, Motivation }\end{array}$ \\
\hline Determination & -0.038 & $1 / 8$ & - & - & - \\
\hline $\begin{array}{l}\text { Emotions } \\
\text { management }\end{array}$ & $-0.151^{* *}$ & $7 / 8$ & $\begin{array}{c}\text { Autonomy, } \\
\text { Confidence at school, } \\
\text { Curiosity, Distrust, } \\
\text { Motivation }\end{array}$ & Autonomy & Autonomy \\
\hline
\end{tabular}


Table 4. Cont.

\begin{tabular}{|c|c|c|c|c|c|}
\hline & \multicolumn{2}{|c|}{ Coefficient of $\mathbf{r}$} & \multicolumn{3}{|c|}{ Control Effect on Inter-Trait Partial } \\
\hline & $\begin{array}{c}\text { Grade } \\
\text { Averages }\end{array}$ & $\begin{array}{l}\text { Number of } \\
\text { Subjects } \\
(p<0.05)\end{array}$ & $\begin{array}{l}\text { Grade Averages } \\
\qquad(p<0.05)\end{array}$ & Gender & $\begin{array}{l}\text { School Cycle (Middle } \\
\text { School-High School) }\end{array}$ \\
\hline Motivation & $0.591 * *$ & $8 / 8$ & $\begin{array}{c}\text { Autonomy, } \\
\text { Confidence at school, } \\
\text { Curiosity, Distrust, } \\
\text { Motivation }\end{array}$ & $\begin{array}{c}\text { Autonomy, } \\
\text { Confidence at school, } \\
\text { Curiosity, Distrust, } \\
\text { Motivation }\end{array}$ & $\begin{array}{c}\text { Autonomy Confidence } \\
\text { at school, Curiosity, } \\
\text { Distrust }\end{array}$ \\
\hline Pleasure & 0.013 & $0 / 8$ & - & - & - \\
\hline
\end{tabular}

The following affective skills: acceptance of critics, autonomy, curiosity, distrust and motivation have (8/8) significant, positive correlations with school subjects' grades represented by a rate of $65.57 \% \%$ of all significant relationships. In general, when controlled by grade averages, gender and school cycle, it is noted that the control effect on intermediate partial correlations is low for each parameter except for curiosity which has an average effect of gender and the school cycle, as well as for motivation, with an average result for the scores.

Additionally, for emotions management, communication and confidence in school present (7/8) significant correlations with school subjects' grades and represent a $34.42 \%$ rate (all significant correlations). Overall, when controlled by grade averages, gender and school cycle, the control effect on inter-trait partial correlations is low for each parameter as regards gender and school cycle, except for school confidence, which has a moderate effect on the average grade level, but low for gender and the school cycle.

The affective parameter: determination presents (1/8) significant correlations with school subjects 'grades and a percentage of $1.63 \%$ (all significant relationships). However, it does not show any control effect on partial inter-trait correlations.

\subsection{Correlation between Educational Outcomes and Cognitive Academic Competencies}

This table shows the correlation between cognitive strategies (analyzing, understanding, focus, distinguish, efficiency, reading and listening attentively, memorizing, taking notes and resolving) and school results.

We noticed that of the 72 correlations calculated, 59 relationships were very significant ( $p=0.009$ to 0.01 ), representing $81.9 \%$ of the relationships. Reading and listening attentively had highly significant correlations $(p=0.00)$.

Cognitive skills have highly significant and positive correlations with grade averages, except for the "resolving" parameter that has no significant correlation. The "memorizing" parameter generates a high intensity. While analyzing, understanding, focus, distinguish, and efficiency parameters show an average intensity. Finally, "taking notes" and "reading and listening attentively" have a low intensity.

The results of the following cognitive skills: analyzing, understanding, focus, distinguish, efficiency and memorizing present (8/8) significant correlations with school subjects' grades, or $81.35 \%$ of all significant relationships. Overall, when controlled by grade averages, gender and school cycle, the control effect on inter-trait partial correlations is low for each parameter as regards gender and school cycle, except for "reading and listening attentively", which has a low effect on the average grade level, but moderate for gender and school cycle.

The attentively reading-listening skill presents (6/8) significant correlations $(10.16 \%$ of all significant correlations). We note at first, that for the grade averages, the cognitive parameters: distinguish, focus and memorizing have a moderate controlled effect of inter-trait partial correlations and low for the analyzing, understanding and efficiency parameters. At gender level, it is noted that the control effect on inter-trait partial correlations is aver- 
age for each cognitive skill except for "efficiency" and "memorizing" which have a high effect. It should be noted that for the school cycle. The following parameters: analyzing, understanding and distinguish have a moderate controlled effect, while focus, efficiency and memorizing parameters generate a high-controlled effect.

The results of cognitive competence show that taking notes presents $(5 / 8)$ significant correlations with school grades ( $8.47 \%$ of all significant relationships) when it is controlled by grade averages, gender and school cycle. The control effect on inter-trait partial correlations is average for each parameter.

\subsection{Correlation between Educational Outcomes and Metacognitive Academic Skills}

The Table 5 below shows the correlation between metacognitive strategies and educational outcomes. It shows the correlation between 5 metacognitive parameters. We noted that out of the 40 correlations calculated, 31 relationships are very significant $(p=0.009$ to 0.01$), 77.5 \%$ of all relationships. Except for "time planification", which had a significant relationship ( $p=0.012$ to 0.04 ).

Table 5. Summary of the partial correlations between cognitive skills controlled by the average effect of grades, gender, and school cycle (middle school, high school).

\begin{tabular}{|c|c|c|c|c|c|}
\hline & \multicolumn{2}{|c|}{ Coefficient of $\mathbf{r}$} & \multicolumn{3}{|c|}{ Control Effect on Inter-Trait Partial } \\
\hline & $\begin{array}{c}\text { Grade } \\
\text { Averages }\end{array}$ & $\begin{array}{c}\text { Number of } \\
\text { Subjects } \\
(p<0.05)\end{array}$ & $\begin{array}{l}\text { Grade Averages } \\
\qquad(p<0.05)\end{array}$ & Gender & $\begin{array}{l}\text { School Cycle (Middle } \\
\text { School-High School) }\end{array}$ \\
\hline Analyzing & $0.279 * *$ & $8 / 8$ & $\begin{array}{l}\text { focus, Distinguish, } \\
\text { Memorizing }\end{array}$ & $\begin{array}{l}\text { Understanding, Focus, } \\
\text { Distinguish, Efficiency, } \\
\text { Memorizing }\end{array}$ & $\begin{array}{l}\text { Understanding, Focus, } \\
\text { Distinguish, Efficiency }\end{array}$ \\
\hline Understanding & $0.352 * *$ & $8 / 8$ & $\begin{array}{l}\text { Memorizing, } \\
\text { taking notes }\end{array}$ & $\begin{array}{c}\text { Concentration, } \\
\text { Distinguish, Efficiency, } \\
\text { Memorize, Taking notes }\end{array}$ & $\begin{array}{c}\text { Analyzing, Focus, } \\
\text { Distinguish, Efficiency, } \\
\text { Memorizing, Taking } \\
\text { notes }\end{array}$ \\
\hline Focus & $0.452 * *$ & $8 / 8$ & $\begin{array}{l}\text { Analyzing, } \\
\text { Distinguish, } \\
\text { Efficiency, Reading } \\
\text { and Listening } \\
\text { Attentively, } \\
\text { Memorizing, } \\
\text { Taking Notes } \\
\end{array}$ & $\begin{array}{c}\text { Analyzing, } \\
\text { Understanding, } \\
\text { Distinguish, Reading } \\
\text { and Listening } \\
\text { Attentively, Memorizing, } \\
\text { Taking notes }\end{array}$ & $\begin{array}{c}\text { Analyzing, } \\
\text { Understanding, } \\
\text { Distinguish, Efficiency, } \\
\text { Reading and listening } \\
\text { attentively, Memorizing, } \\
\text { Taking notes }\end{array}$ \\
\hline Distinguish & $0.403 * *$ & $8 / 8$ & $\begin{array}{l}\text { Analyzing, focus, } \\
\text { Memorizing, } \\
\text { Taking notes }\end{array}$ & $\begin{array}{c}\text { Analyzing, } \\
\text { Understanding, Focus, } \\
\text { Efficiency, Memorizing, } \\
\text { Taking notes }\end{array}$ & $\begin{array}{c}\text { Analyzing, } \\
\text { Understanding, Focus, } \\
\text { Efficiency, Memorizing, } \\
\text { Taking notes }\end{array}$ \\
\hline Efficiency & $0.568 * *$ & $8 / 8$ & $\begin{array}{l}\text { Focus, Taking } \\
\text { notes }\end{array}$ & $\begin{array}{c}\text { Analyzing, } \\
\text { Understanding, Focus, } \\
\text { Distinguish, Reading } \\
\text { and Listening } \\
\text { Attentively, Memorizing, } \\
\text { Taking Notes }\end{array}$ & $\begin{array}{c}\text { Analyzing, } \\
\text { Understanding, Focus, } \\
\text { Distinguish, Reading } \\
\text { and Listening } \\
\text { Attentively, Memorizing, } \\
\text { Taking notes }\end{array}$ \\
\hline $\begin{array}{l}\text { Reading and } \\
\text { Listening } \\
\text { Attentively }\end{array}$ & $0.126^{* * *}$ & $6 / 8$ & Focus & $\begin{array}{l}\text { Focus, Efficiency, } \\
\text { Memorizing }\end{array}$ & $\begin{array}{l}\text { Focus, Efficiency, } \\
\text { Memorizing }\end{array}$ \\
\hline
\end{tabular}


Table 5. Cont.

\begin{tabular}{|c|c|c|c|c|c|}
\hline & \multicolumn{2}{|c|}{ Coefficient of $r$} & \multicolumn{3}{|c|}{ Control Effect on Inter-Trait Partial } \\
\hline & $\begin{array}{c}\text { Grade } \\
\text { Averages }\end{array}$ & $\begin{array}{c}\text { Number of } \\
\text { Subjects } \\
(p<0.05)\end{array}$ & $\begin{array}{l}\text { Grade Averages } \\
\qquad(p<0.05)\end{array}$ & Gender & $\begin{array}{l}\text { School Cycle (Middle } \\
\text { School-High School) }\end{array}$ \\
\hline Memorizing & $0.904^{* *}$ & $8 / 8$ & $\begin{array}{l}\text { Analyzing, } \\
\text { Understanding, } \\
\text { Focus, Distinguish, } \\
\text { Taking note }\end{array}$ & $\begin{array}{l}\text { Analyzing, } \\
\text { Understanding, Focus, } \\
\text { Distinguish, Efficiency, } \\
\text { Reading and Listening } \\
\text { Attentively, Taking notes }\end{array}$ & $\begin{array}{c}\text { Analyzing, } \\
\text { Understanding, Focus, } \\
\text { Distinguish, Efficiency, } \\
\text { Reading and Listening } \\
\text { Attentively, Taking notes }\end{array}$ \\
\hline Taking notes & $0.109 * *$ & $5 / 8$ & $\begin{array}{l}\text { Understanding, } \\
\text { Distinguish, focus, } \\
\text { Reading and } \\
\text { Listening } \\
\text { Attentively, } \\
\text { Efficiency, } \\
\text { Memorizing }\end{array}$ & $\begin{array}{l}\text { Understanding, } \\
\text { Distinguish, Efficiency, } \\
\text { Memorizing }\end{array}$ & $\begin{array}{c}\text { Understanding, Focus, } \\
\text { Distinguish, Efficiency, } \\
\text { Memorizing. }\end{array}$ \\
\hline
\end{tabular}

\begin{tabular}{llll}
\hline Resolving & 0.010 & - & -
\end{tabular}

The efficiency search strategy (Table 6) has highly significant and positive correlations with grade averages, it has (8/8) significant correlations with grades of school subjects, or about $25.80 \%$ of all relationships. Generally, when controlled by grade averages, gender and school cycle, it is noted that the control effect on inter-trait partial correlations is low for grade averages, gender and school cycle.

The results of the following metacognitive skills: inhibition of distractors, affairs and time organization and cognitive regulation present correlations $(7 / 8)$ with grades of school subjects for each $67.74 \%$ of the significant results: When controlled by grade averages, gender and school cycle, the control effect on inter-trait partial correlations is low for grade averages and means for gender and school cycle.

Time planification presents $(2 / 8)$ correlations or $6,45 \%$ of the significant correlations; that is, the control effect on the inter-trait partial correlations is almost non-existent.

Table 6. Summary of the partial correlations between metacognitive skills controlled by the average effect of grades, gender, and school cycle (middle school, high school).

\begin{tabular}{|c|c|c|c|c|c|}
\hline & \multicolumn{2}{|c|}{ Coefficient of $\mathbf{r}$} & \multicolumn{3}{|c|}{ Control Effect on Inter-Trait Partial } \\
\hline & $\begin{array}{c}\text { Grade } \\
\text { Averages }\end{array}$ & $\begin{array}{l}\text { Number of } \\
\text { Subjects } \\
(p<0.05)\end{array}$ & $\begin{array}{l}\text { Grade Averages } \\
(p<0.05)\end{array}$ & Gender & $\begin{array}{l}\text { School Cycle (Middle } \\
\text { School-High School) }\end{array}$ \\
\hline $\begin{array}{l}\text { Efficiency search } \\
\text { strategy }\end{array}$ & $0.755 * *$ & $8 / 8$ & $\begin{array}{l}\text { Affairs and time } \\
\text { organization, Time } \\
\text { planification }\end{array}$ & $\begin{array}{c}\text { Inhibition of distractors, } \\
\text { Affairs and time } \\
\text { organization, Cognitive } \\
\text { regulation }\end{array}$ & $\begin{array}{c}\text { Inhibition of distractors, } \\
\text { Affairs and time } \\
\text { organization, Time } \\
\text { planification, Cognitive } \\
\text { regulation }\end{array}$ \\
\hline $\begin{array}{l}\text { Inhibition of } \\
\text { distractors }\end{array}$ & $0.209 * *$ & $7 / 8$ & $\begin{array}{l}\text { Cognitive } \\
\text { Regulation }\end{array}$ & $\begin{array}{c}\text { Efficiency search } \\
\text { strategy Affairs and time } \\
\text { organization, Cognitive } \\
\text { regulation }\end{array}$ & $\begin{array}{l}\text { Efficiency search } \\
\text { strategy, Affairs and } \\
\text { time organization, } \\
\text { Cognitive regulation }\end{array}$ \\
\hline $\begin{array}{l}\text { Affairs and time } \\
\text { organization }\end{array}$ & $0.344^{* *}$ & $7 / 8$ & $\begin{array}{l}\text { Efficiency search } \\
\text { strategy }\end{array}$ & $\begin{array}{c}\text { Efficiency search } \\
\text { strategy, Inhibition of } \\
\text { distractors, Cognitive } \\
\text { regulation }\end{array}$ & $\begin{array}{c}\text { Efficiency search } \\
\text { strategy, Inhibition of } \\
\text { distractors, Cognitive } \\
\text { regulation }\end{array}$ \\
\hline
\end{tabular}


Table 6. Cont.

\begin{tabular}{cccccc}
\hline & \multicolumn{2}{c}{ Coefficient of $\mathbf{r}$} & \multicolumn{2}{c}{ Control Effect on Inter-Trait Partial } \\
\hline Averages & $\begin{array}{c}\text { Number of } \\
\text { Subjects } \\
(p<\mathbf{0 . 0 5})\end{array}$ & $\begin{array}{c}\text { Grade Averages } \\
(p<\mathbf{0 . 0 5})\end{array}$ & Gender & $\begin{array}{c}\text { School Cycle (Middle } \\
\text { School-High School) }\end{array}$ \\
\hline $\begin{array}{c}\text { Time } \\
\text { planification }\end{array}$ & $0.074^{*}$ & $2 / 8$ & - & - & - \\
\hline $\begin{array}{c}\text { Cognitive } \\
\text { regulation }\end{array}$ & $0.152 * *$ & $7 / 8$ & $\begin{array}{c}\text { Inhibition of } \\
\text { distractors, Affairs } \\
\text { and time } \\
\text { organization }\end{array}$ & $\begin{array}{c}\text { Efficiency search } \\
\text { strategy, Inhibition of } \\
\text { distractors, Time }\end{array}$ & $\begin{array}{c}\text { Efficiency search } \\
\text { strategy, Inhibition of } \\
\text { distractors, Time } \\
\text { time organization }\end{array}$ \\
\hline
\end{tabular}

** Very significant $(p=0.009$ to 0.01$) ; *$ Significant $(p=0.012$ to 0.04$)$.

\section{Discussion}

Let us remember, first of all, that our research aims to study the correlation between the psychological characteristics of the student's academic behavior that translate in terms of personality traits and mental skills with his academic results. It aims to identify the personality traits and learning strategies adopted by the student by explaining his school performance. We will discuss the main results in the light of the objective of research: to study the correlation between the psychological characteristics of the student's academic behavior in terms of personality traits and mental skills, with his academic results.

Our first results showed that all dimensions of personality have significant relationships, citing the main ones such as abstractedness, tension, emotional stability, dominance, social-boldness, vigilance and apprehension. These have an impact on school grades and these relationships are highly significant overall with low intensity. This shows that confident, open-minded, motivated, carefree, dominant, assertive and competitive students are statistically able to increase their school grades.

These results were confirmed by Almlund et al. [24], who demonstrated that students' performances and academic achievements depend on their personality traits, having a significant effect. For example, consciousness has been fundamentally positively related to educational outcomes [25,26], meanwhile Chamorro-Premuzic and Furnham [27] mentioned that cognitive abilities and educational level are regularly correlated by openness to change and awareness. In addition to that, Costa et al. [28] and Schmitt et al. [29] have explicated this difference in relation to gender and the learning context.

The proposed assumptions were fully confirmed by the results. Psycho-pedagogically speaking, the teacher must learn about the psychological level of the population he faces. If the active theories of learning are highlighted in teaching that focuses on the needs and expectations of students and that contextual aspect (socio-economic, demographic characteristics, etc.) must be considered, it is essential to detect these personality traits to better understand the context to which they belong and to better act and interact. The idea is to understand what motivates them and to maintain their active engagement in order to offer coaching lessons in the context of a particular situation or to refer them to a specialist.

All these efforts are for student interest and have a significant influence on his or her academic results. Educational sociologists have insisted on making efforts at the level of learning social group because this parameter allows students to develop the primary social skills. For this reason, it seems appropriate for the teacher at a learning level to form groups according to their psychological profiles; this could promote the synergy of this group and eventually an increase in the collective and individual students' performances.

However, mixed results emerged for the relationships between pupil extroversion and academic success, ranging from zero outcomes [30] to negative associations [31,32]. Adding to this, other studies have not reported any differences between gender, openness to change 
and consciousness [28]. Differences in suitability between the last ones are systematically replicated in studies where women's scores are higher than men's [28,33].

The results showed that there is a significant influence of personality on school grades even though the limits of our approach seem to be mentioned. Statistically speaking, it was better to work in different contexts and not focus on one in particular (the case of the Mediouna provincial directorate for our study), in order to give more reliability to our sample [34] and avoid selection bias. This way, we can widen it with elementary and university students.

Following the results, we propose to:

- Identify student personality traits and relate them to teaching styles and animations techniques.

- To deepen much more on this study by proposing a modeling of personality traits promoting academic success according to age, school cycle (primary, middle-school and high- school), gender etc.

The results show that all the affective strategies assessed have a highly significant relationship with school grades, except for determination and pleasure, from a statistical point of view. The main results were acceptance of errors and critics, autonomy, curiosity, distrust, emotions management and motivation scored the highest rates. That said, these skills provide the student with an optimal emotional state to carry out the tasks requested.

It has been validated by Bryan et al. [35] and also Ryan and Patrick [36] that academic motivation is well established as an important factor related to student performance in the school environment. The research identified the link between student motivation and engagement in school activities, such as studying, participating in classroom discussions, and working with peers under several circumstances [37].

The cognitive mental skills results show that analyzing, understanding, focus, distinguish, efficiency and memorizing are the main strategies of cognitive learning promoting positive school performances. Moreover, students who have developed these skills are more likely to obtain good grades. It is confirmed by Hattie, Biggs and Purdie [38], Murayama et al. [39] and Nota et al. [40] that students can control their learning process by using learning strategies and therefore positively influence their academic outcomes.

Related to metacognitive skills, effective strategic research, inhibition, organization and cognitive regulation present the main metacognitive strategies. It is, in fact, metacognitive thoughts and behaviors mobilized to achieve a task effectively. A recent meta-analysis by Dent and Koenka [41] proved that using metacognitive strategies can increase student achievement by $4 \%$. Additionally, Dunning et al. [42] confirm that the ability to demonstrate metacognitive awareness and employ metacognitive strategies contributes significantly to academic outcomes. It can be concluded that difference in educational level would probably have an impact in their engagement in metacognitive strategies [43-46].

Referring to assumptions that have been confirmed, learning strategies are used to identify all behaviors adopted by student. Interest in learning strategies is supported by active learning theories. Respecting the concept of skill, students are expected to use these strategies to achieve the skill, which is characterized by its junction dimension. It is consistent with the current conception of learning as a complex and dynamic process involving cognitive, metacognitive, motivational and emotional dimensions in reciprocal and dynamic interaction. From a didactic point of view, the teacher's role resides in the ability to appreciate the psychological and mental consequences of these teaching methods adopted independently of the students' academic performance since this is not a reliable criterion. Therefore, there is a need to question these professional practices.

However, performance situations, knowledge generation or task execution are not considered by learning strategies taxonomies [47]. Indeed, questionnaires or interviews promote an introspective aspect to their answers, with reflexive look at their learning methods which he might not have done spontaneously [19]. On the other hand, positive school results are linked by controlled motivation according to several parameters, but could also negatively affect performance when controlled incentives are given and the 
quality of performance is assessed [37]. Nevertheless, no significant relationships could be found in other studies, either among under-graduated students [48-51] or among high school students [52].

In a larger study in Hong Kong including 8873 students that examined the relationship between purposes, metacognition and academic achievement, it was found that there is a solid association with purposes and metacognition. However, achievement had no significant relationship [53]. It means that metacognitive strategies are used by high-achieving students even if the relationship of these three variables is related, but not reciprocal.

The results showed a significant influence of mental skills in grades averages, although a major limitation of our research needs to be mentioned: using an unvalidated scale measuring academic mental skills affects the reliability of our results. In fact, Rouder et al. [54] confirm that studies of individual differences using tasks with low reliability is ineffective. Additionally, it was desirable to correlate between each learning strategy with each subject. However, because of time, we could not realize it. Research in this direction must therefore be carried out in several contexts for more exhaustivity of our research.

We may then propose to:

- Model learning strategies according to the requirements of each teaching situation proposed to the student.

- Link all learning strategies involved in each activity for each school subject. As well as classify them according to school level.

\section{Conclusions}

The purpose of our study was to correlate the personality traits and students' mental skills with their academic achievement for a relevant and effective pedagogical intervention. Thus, it was essential to start with the distribution of the 16PF5 test, the mental skills assessment scale.

Through this study, we have been able to get results showing that the personality traits identified fully confirmed the relationship between personality traits and educational outcomes - that it is a highly significant relationship. It shows that the psychological aspect is important to better understanding student behavior and to give them the right psychological support.

After that, the affective, cognitive and metacognitive learning strategies proved their relevance in terms of educational outcomes, as they promote positive academic outcomes. Learning strategies allow the understanding of the complexity of learner mental behaviors. Therefore, it seems essential to provide more effort in order to give more efficiency and relevance to teacher's intervention.

Certainly, the act of teaching remains a complex process requiring the intervention of several factors to bring the students the necessary skills. The results obtained are a step towards a more in-depth vision on the part of all the actors of the Moroccan education system. The psychological dimension is a factor to be taken into consideration for an effective and relevant educational intervention, as the student lives in a stimulating context that impacts his psychic balance and allows him to mobilize internal and external resources. This should be the teacher's priority. Altet [55] introduces the concept of professional practice analysis questioning the teacher's pedagogical practice, which elicits broader reflection.

Based on this conclusion, to what extent can we consider the correlation between each learning strategy and each school subject?

In order to increase the impact of our study, we propose:

- The development of a system to assist students' families in precarious situations in order to enlighten them on the practices improving the student profile. These projects must be carried out with the Ministry of National Education and the Ministry of Family and Solidarity. For the same purpose, it is also possible to set up another system based on the aims of physical and sports education in agreement with the Ministry of Youth and Sport. 
- The skills reference frame should be focused on the impact of student's personality traits on school performance with the need to recruit future teachers to consider these parameters when setting up work.

- Link personality traits and learning strategies with academic scores to understand the student's interaction with subjects and their excellence in a particular relative to others.

- Correlate learning strategies with each school subject, which involves work by didactic experts in order to facilitate the task of teachers, on the one hand, and making the subject more accessible to all students on the other hand.

- Develop a modeling of each learning strategy (affective, cognitive and metacognitive) based on the taxonomies of learning situations for relevance in the choice of learning activities.

- Review the teacher competency framework by adding a personality dimension according to the psychological profile of the students to answer the question: which teacher for which student? A question that is supported in our analysis of the correlation between the student's personality and his school grades, we thought it would be appropriate to add the teacher profile for more completeness of our analysis.

- Try to correlate learning strategies and student guidance to encourage students to make better choices based on their own mental skills and competencies.

Author Contributions: Conceptualization, S.L. and O.B.; methodology, S.L. and M.T.; software, S.L.; validation, S.L., M.T. and O.B.; formal analysis, S.L.; investigation, O.B.; resources, O.B.; data curation, O.B.; writing—original draft preparation, O.B.; writing—review and editing, O.B.; visualization, S.L.; supervision, S.L. and M.T.; project administration, M.T. and S.L. All authors have read and agreed to the published version of the manuscript.

Funding: This research received no external funding.

Institutional Review Board Statement: Ethical review and approval were waived for this study. In fact, one of the authors is a physical education teacher. Therefore, there is no problem because he has the right to do this kind of research on his own student.

Informed Consent Statement: Informed consent was obtained from all subjects involved in the study.

Data Availability Statement: The data presented in this study are available on request from the corresponding author. The data are not publicly available due to confidential reasons.

Acknowledgments: We would like to thank all the students and their teachers for their availability and implication during data collection.

Conflicts of Interest: The authors declare no conflict of interest.

\section{References}

1. Bandura, A. Much Ado over a Faulty Conception of Perceived Self-Efficacy Grounded in Faulty Experimen-tation. J. Soc. Clin. Psychol. 2007, 26, 641-658. [CrossRef]

2. Alexander, K.L.; Entwisle, D.R.; Dauber, S.L. First-Grade Classroom Behavior: Its Short- and Long-Term Consequences for School Performance. Child Dev. 1993, 64, 801. [CrossRef]

3. Florin, A.; Guimard, P.; Nocus, I. Les Évaluations Des Enseignants et La Prédiction Des Compétences Lan-gagières de Leurs Élèves: Études Longitudinales à l'école Maternelle et à l'école Élémentaire. Le Lang. Et L'homme 2002, 2, 175-190.

4. Guimard, P.; Cosnefroy, O.; Florin, A. Évaluation des comportements et des compétences scolaires par les enseignants et prédiction des performances et des parcours à l'école élémentaire et au collège. Orientat. Sc. Prof. 2007, 36, 179-202. [CrossRef]

5. McClelland, M.M.; Morrison, F.J.; Holmes, D.L. Children at Risk for Early Academic Problems: The Role of Learning-Related Social Skills. Early Child. Res. Q. 2000, 15, 307-329. [CrossRef]

6. McClelland, M.M.; Acock, A.C.; Morrison, F.J. The Impact of Kindergarten Learning-Related Skills on Ac-ademic Trajectories at the End of Elementary School. Early Child. Res. Q. 2006, 21, 471-490. [CrossRef]

7. Yen, C.-J.; Konold, T.R.; McDermott, P.A. Does Learning Behavior Augment Cognitive Ability as an Indicator of Academic Achievement? J. Sch. Psychol. 2004, 42, 157-169. [CrossRef]

8. Chamarro-Premuzic, T.; Furnham, A. Personality Traits and Academic Examination Performance. Eur. J. Pers. 2003, 17, 237-250. [CrossRef] 
9. Furnham, A.; Chamorro-Premuzic, T.; McDougall, F. Personality, Cognitive Ability, and Beliefs about Intelli-gence as Predictors of Academic Performance. Learn. Individ. Differ. 2003, 14, 47-64. [CrossRef]

10. Poropat, A.E. A Meta-Analysis of the Five-Factor Model of Personality and Academic Performance. Psychol. Bull. 2009, 135, 322-338. [CrossRef] [PubMed]

11. Loret, J.M. Estilos y Estrategias de Aprendizaje En El Rendimiento Académico de Los Estudiantes de La Universidad Peruana "Los Andes" de Huancayo. Perú. Rev. Estilos Aprendiz. 2011, 8, 8.

12. Barca-Lozano, A.; Peralbo, M.; Almeida, L.S.; Brenlla, J.C.; Vicente, F.; Porto, A.; Morán, H. Learning Strategies and Academic Achievement. Efectos Metas Académicas 2011, 165, 3047-3077.

13. Tejedor-Tejedor, F.J.; González Salvador, G.; García Señorál, M.M. Estrategias Atencionales y Rendimiento Académico En Estudiantes de Secundaria. Rev. Lat. 2008, 40, 123-132.

14. Boulet, A.; Savoie-Zajc, L.; Chevrier, J. Les Stratégies D'apprentissage à L'université; Presse de l’Université du Québec: Sainte-Foy, France, 2008.

15. Fayol, M.; Monteil, J.-M. Note de synthèse [Stratégies d'apprentissage/apprentissage de stratégies]: Stratégies d'apprentissage/ apprentissage de stratégies. Rev. fr. Pédagog. 1994, 106, 91-110. [CrossRef]

16. Lapointe, F.; Bernard, H.; Frenay, M.; Noël, B.; Parmentier, P.; Romainville, M. L'étudiant-Apprenant, Grille de Lecture Pour l'enseignant Universitaire. Paris/Bruxelles: De Boeck Université (Collection «Perspectives En Éducation»). Rev. Sci. Edu. 2001, $27,233$.

17. Hrimech, M. Les Stratégies d'apprentissage En Contexte d'autoformation. Dans 2000, 34, 99-111.

18. Langevin, L. Stratégies d'apprentissage: Où En Est La Recherche. Vie Pédagogique 1992, 77, $39-43$.

19. Turgeon, M.; Romainville, M. Savoir parler de ses méthodes. Métacognition et performance à l'université. Bruxelles: De Boeck Université. Rev. Sci. Edu. 1994, 20, 408.

20. Weinstein, E.; Ellen, C.; Hume, L. Stratégies Pour Un Apprentissage Durable; De Boeck Université: Paris, France, 2001.

21. Cresswell, J.; Underwood, C. Location, Location, Location; Australian Council for Educational Research Ltd.: Camberwell, Australia, 2004. Available online: https:/ / core.ac.uk/download/pdf/36780197.pdf (accessed on 11 June 2020).

22. Rothman, S.; McMillan, J. Influences on Achievement in Literacy and Numeracy; Longitudinal Survey of Australian Youth Australian Council for Educational Research: Camberwell, Australia, 2003. Available online: https://research.acer.edu.au/cgi/viewcontent. cgi?article=1039\&context=lsay_research (accessed on 13 May 2020).

23. Bourqia, R. Repenser et Refonder l'école Au Maroc: La Vision Stratégique 2015-2030. Rev. Int. Éduc. Sèvres 2016, 71, 18-24. [CrossRef]

24. Almlund, M.; Lee Duckworth, A.; Heckman, J.J.; Kautz, T. Personality Psychology and Economics; Hanushek, E.A., Machin, S., Woessmann, L., Eds.; Elsevier: Amsterdam, The Netherlands, 2011; pp. 1-181.

25. Chamorro-Premuzic, T.; Furnham, A. Personality Predicts Academic Performance: Evidence from Two Lon-gitudinal Studies on British University Students. J. Res. Personal. 2003, 37, 319-338. [CrossRef]

26. Spengler, M.; Brunner, M.; Martin, R.; Lüdtke, O. The Role of Personality in Predicting (Change in) Students' Academic Success across Four Years of Secondary School. Eur. J. Psychol. Assess. 2016, 32. [CrossRef]

27. Chamorro-Premuzic, T.; Furnham, A. Personality and Intelligence as Predictors of Statistics Examination Grades. Personal. Individ. Differ. 2004, 37, 943-955.

28. Costa, P.T.; Terracciano, A.; McCrae, R.R. Gender Differences in Personality Traits across Cultures: Robust and Surprising Findings. J. Pers. Soc. Psychol. 2001, 81, 322-331. [CrossRef] [PubMed]

29. Schmitt, D.P.; Realo, A.; Voracek, M.; Allik, J. Why Can't a Man Be More like a Woman? Sex Differences in Big Five Personality Traits across 55 Cultures. J. Personal. Soc. Psychol. 2008, 94, 168-182. [CrossRef] [PubMed]

30. Laidra, K.; Pullmann, H.; Allik, J. Personality and Intelligence as Predictors of Academic Achievement: A Cross-Sectional Study from Elementary to Secondary School. Personal. Individ. Differ. 2007, 42, 441-451. [CrossRef]

31. Lechner, C.; Danner, D.; Rammstedt, B. How Is Personality Related to Intelligence and Achievement? A Replication and Extension of Borghans et Al. and Salkever. Personal. Individ. Differ. 2017, 111, 86-91. [CrossRef]

32. O'Connor, M.C.; Paunonen, S.V. Big Five Personality Predictors of Post-Secondary Academic Perfor-mance. Personal. Individ. Differ. 2007, 43, 971-990. [CrossRef]

33. Weisberg, Y.J.; DeYoung, C.G.; Hirsh, J.B. Gender Differences in Personality across the Ten Aspects of the Big Five. Front. Psychol. 2011, 2, 178. [CrossRef] [PubMed]

34. Questions à Philippe Blanchet. Cah. Socioling 2005, 10, 48.

35. Bryan, R.R.; Glynn, S.M.; Kittleson, J.M. Motivation, Achievement, and Advanced Placement Intent of High School Students Learning Science. Sci. Educ. 2011, 95, 1049-1065. [CrossRef]

36. Ryan, A.M.; Patrick, H. The Classroom Social Environment and Changes in Adolescents' Motivation and Engagement During Middle School. Am. Educ. Res. J. 2001, 38, 437-460. [CrossRef]

37. Cerasoli, C.P.; Nicklin, J.M.; Ford, M.T. Intrinsic Motivation and Extrinsic Incentives Jointly Predict Per-formance: A 40-Year Meta-Analysis. Psychol. Bull. 2014, 140, 980-1008. [CrossRef]

38. Hattie, J.; Biggs, J.; Purdie, N. Effects of Learning Skills Interventions on Student Learning: A Me-ta-Analysis. Rev. Educ. Res. 1996, 66, 99-136. [CrossRef] 
39. Murayama, K.; Pekrun, R.; Lichtenfeld, S.; Vom Hofe, R. Predicting Long-Term Growth in Students' Mathe-matics Achievement: The Unique Contributions of Motivation and Cognitive Strategies. Child Dev. Ment. 2013, 84, 1475-1490. [CrossRef]

40. Nota, L.; Soresi, S.; Zimmerman, B.J. Self-Regulation and Academic Achievement and Resilience: A Longitu-dinal Study. Int. J. Educ. Res. 2004, 41, 198-215. [CrossRef]

41. Dent, A.L.; Koenka, A.C. The Relation 1 between Self-Regulated Learning and Academic Achievement across Childhood and Adolescence: A Meta-Analysis. Educ. Psychol. Rev. 2016, 28, 425-474. [CrossRef]

42. Dunning, D.; Johnson, K.; Ehrlinger, J.; Kruger, J. Why People Fail to Recognize Their Own Incompe-tence. Curr. Dir. Psychol. Sci. 2003, 12, 83-87. [CrossRef]

43. Boekaerts, M. Motivation and Self-Regulation: Two Close Friends; Urdan, T.C., Karabenick, S.A., Eds.; Emerald: Bingley, UK, 2010; pp. 69-108.

44. Boekaerts, M.; Koning, E.; Vedder, P. Goal-Directed Behavior and Contextual Factors in the Classroom: An Innovative Approach to the Study of Multiple Goals. Educ. Psychol. 2006, 41, 33-51. [CrossRef]

45. Zimmerman, B.J. From Cognitive Modeling to Self-Regulation: A Social Cognitive Career Path. Educ. Psychol. 2013, 48, 135-147. [CrossRef]

46. Zimmerman, B.J.; Schunk, D.H. Motivational Sources and Outcomes of Self-Regulated Learning and Per-Formance; Schunk, D.H., Zimmerman, B., Eds.; Taylor \& Francis: New York, NY, USA, 2011; pp. 49-64.

47. Towns, M.H.; Robinson, W.R. Student Use of Test-Wiseness Strategies in Solving Multiple-Choice Chemistry Examinations. J. Res. Sci. Teach. 1993, 30, 709-722. [CrossRef]

48. Muis, K.R.; Pekrun, R.; Sinatra, G.M.; Azevedo, R.; Trevors, G.; Meier, E.; Heddy, B.C. The Curious Case of Climate Change: Testing a Theoretical Model of Epistemic Beliefs, Epistemic Emotions, and Complex Learning. Learn. Instr. 2015, 39, 168-183. [CrossRef]

49. Pekrun, R.; Goetz, T.; Titz, W.; Perry, R.P. Academic Emotions in Students' Self-Regulated Learning and Achievement: A Program of Qualitative and Quantitative Research. Educ. Psychol. 2002, 37, 91-105. [CrossRef]

50. Pekrun, R.; Goetz, T.; Daniels, L.M.; Stupnisky, R.H.; Perry, R.P. Boredom in Achievement Settings: Exploring Control-Value Antecedents and Performance Outcomes of a Neglected Emotion. J. Educ. Psychol. 2010, 102, 531-549. [CrossRef]

51. Pekrun, R.; Goetz, T.; Frenzel, A.C.; Barchfeld, P.; Perry, R.P. Measuring Emotions in Students' Learning and Performance: The Achievement Emotions Questionnaire (AEQ). Contemp. Educ. Psychol. 2011, 36, 36-48. [CrossRef]

52. King, R.B.; Areepattamannil, S. What Students Feel in School Influences the Strategies They Use for Learning: Academic Emotions and Cognitive/Meta-Cognitive Strategies. J. Pac. Rim Psychol. 2014, 8, 18-27. [CrossRef]

53. King, R.B.; McInerney, D.M. Do Goals Lead to Outcomes or Can It Be the Other Way around? Causal Ordering of Mastery Goals, Metacognitive Strategies, and Achievement. Br. J. Educ. Psychol. 2016, 86, 296-312. [CrossRef]

54. Rouder, J.N.; Kumar, A.; Haaf, J.M. Why Most Studies of Individual Differences with Inhibition Tasks Are Bound to Fail. PsyArXiv 2019. [CrossRef]

55. Altet, M. La Formation Professionnelle Des Enseignants; Presses Universitaires de France: Paris, France, 1994. 